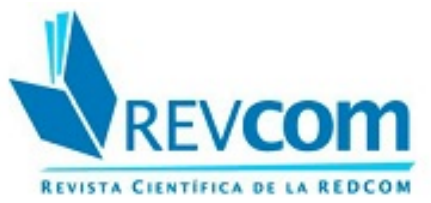

REVCOM. Revista científica de la red de carreras de Comunicación Social

ISSN: 2451-7836

redcom.revcom@gmail.com

Universidad Nacional de La Plata

Argentina

\title{
Encuesta sobre apropiación de tecnologías por parte de niños y niñas de 6 a 8 años
}

Cabello, Roxana; Alonso, Juan Manuel

Encuesta sobre apropiación de tecnologías por parte de niños y niñas de 6 a 8 años

REVCOM. Revista científica de la red de carreras de Comunicación Social, núm. 9, 2019

Universidad Nacional de La Plata, Argentina

DOI: https://doi.org/10.24215/24517836e025 


\section{Encuesta sobre apropiación de tecnologías por parte de niños y niñas de 6 a 8 años}

Pesquisa sobre apropriação de tecnologias por meninos e meninas de 6 a 8 anos

Roxana Cabello

DOI: https://doi.org/10.24215/24517836e025

Universidad Nacional de General Sarmiento, Argentina

rcabello@campus.ungs.edu.ar

Juan Manuel Alonso

Universidad Nacional de General Sarmiento, Argentina

Recepción: 14 Agosto 2019

jmalonso@campus.ungs.edu.ar

\section{Resumen:}

En el Programa Usos de Medios Interactivos (UMI) de la Universidad Nacional de General Sarmiento (UNGS) en Argentina, estamos desarrollando el "Estudio de procesos de apropiación de tecnologías por parte de niños y niñas de 6 a 8 años de edad". Buscamos comprender las características que asumen esos procesos en un período de la infancia en el que se construyen las competencias de lectura y escritura. Presentamos en este artículo algunos resultados obtenidos a través de una encuesta a personas adultas a cargo de niños y niñas de ese tramo de edad. Abordamos tres dimensiones que adjudicamos teóricamente a la noción de hogar como entorno tecnocultural: disposición de tecnologías, dinámica de usos de tecnologías en el hogar y regulaciones sobre los usos de tecnologías. Comentamos aquí únicamente los usos de tecnologías (sobre todo internet) que, según las personas adultas, realizan los niños y las niñas en el contexto del hogar.

Palabras CLAVE: Investigación, apropiación, tecnologías, niños y niñas.

\section{Resumo:}

No Programa de Uso de Mídia Interativa (UMI) do UNGS na Argentina, estamos desenvolvendo o "Estudo dos procesos da apropriaçao de tecnología por crianças de 6 a 8 anos de idade”. Buscamos compreender as características desses processos em um período de vida em que as habilidades de leitura e escrita são construídas. Apresentamos neste artigo alguns resultados obtidos através de uma pesquisa com adultos encarregados de crianças dessa faixa etária. Abordamos três dimensões que, teoricamente, atribuímos à noção de lar como um ambiente tecnocultural: disposiçâo de tecnologias, dinâmica de usos de tecnologia em casa e regulamentos sobre os usos das tecnologias. Comentamos aqui apenas sobre os usos das tecnologias (especialmente a Internet) que, de acordo com os adultos, as crianças fazem no contexto do lar.

PalaVRas-CHAVE: Investigação, apropriação, tecnologias, crianças.

\section{INTRODUCCIÓN}

En la actualidad, algunos discursos tienden a naturalizar la relación que los niños y las niñas establecen con los dispositivos técnicos y con internet. Sobre todo a través de los medios de comunicación se transmite la idea de que, como crecen rodeados de tecnologías y las usan a edades cada vez más tempranas, se familiarizan e incorporan "naturalmente" unos saberes y habilidades que las personas adultas no tienen o demoran mucho en construir.

En el Programa Usos de Medios Interactivos (UMI) del Área de Comunicación de la Universidad Nacional de General Sarmiento, en Argentina, investigamos desde hace casi veinte años sobre los vínculos que las personas establecemos con las tecnologías. De acuerdo con nuestra experiencia, la edad es solamente una de las variables que interviene diferencialmente en los procesos de apropiación de tecnologías y está, en general, asociada a otras como el nivel socioeconómico y el clima educativo del hogar ${ }^{1}$. Algunas de esas relaciones son las que estamos investigando a través del proyecto "Estudio de procesos de apropiación de 
tecnologías por parte de niños y niñas de 6 a 8 años de edad”. Entendemos a la apropiación de tecnologías desde una perspectiva socio-histórica (López, 2017) como un proceso social de aprendizaje que el sujeto realiza a través de actividades medidas por artefactos técnicos (incluyendo procesos, lenguajes, productos) y de sus propias vivencias (involucrando entonces no solamente aspectos cognitivos sino emocionales). Este proceso se produce siempre de manera situada en contextos socio culturales en los cuales se participa a partir de desiguales condiciones, y contempla distintos aspectos entre los cuales enfocamos: el acceso y disponibilidad de tecnologías, los usos que se hacen de ellas, los conocimientos asociados (sobre, con y a partir de) con ellas, las competencias vinculadas con sus usos, la capacidad de objetivarlas y analizarlas críticamente. A través de un complejo diseño cualitativo y cuantitativo, buscamos comprender las características que asumen esos procesos en un período de la vida en el que se construyen las competencias comunicativas de lectura y escritura. Dado que a esas edades se utilizan más frecuentemente los dispositivos móviles (teléfono celular y tableta), abordamos dos ámbitos que tienen mayor estabilidad: el hogar y la escuela, entendidos como entornos tecnoculturales en el área metropolitana de Buenos Aires. Por un lado, realizamos con los niños y niñas talleres de juegos e internet, observaciones, actividades gráficas y entrevistas grupales. Por otro lado, desarrollamos la "Encuesta sobre apropiación de tecnologías en niños y niñas de 6 a 8 años de edad" y presentamos en este artículo algunos de los resultados obtenidos. A través de la encuesta abordamos las tres dimensiones que adjudicamos a la noción de hogar como entorno tecnocultural: disposición de tecnologías, dinámica de usos de tecnologías en el hogar y regulaciones sobre los usos de tecnologías. Comentamos aquí únicamente los usos de tecnologías (sobre todo internet) que, según las personas adultas, realizan los niños y las niñas en el contexto del hogar. El trabajo de campo se realizó entre septiembre y octubre de 2018 (en el marco del Seminario Usos Sociales de las Tecnologías, UNGS), mediante técnica de recolección personal ("cara a cara"), con cuestionario directo y semi-estructurado aplicado a una muestra intencional ("bola de nieve”) de 357 hogares ubicados en 12 partidos del conurbano bonaerense (Gran Buenos Aires). Se entrevistó a personas adultas a cargo de niños y niñas de 6 a 8 años de edad.

En los que respecta a los usos de tecnologías, enfocamos en este artículo datos sobre acceso y conectividad (cotejados con estudios realizados a nivel nacional), tiempo destinado a los usos de internet y preferencias. Realizamos algunos comentarios sobre los usos principales de internet: Youtube y juegos en línea y también sobre algunos aspectos relacionados con esas variables que, ratificamos, condicionan la relación que lxs chicxs establecen con las tecnologías.

\section{El acceso a internet en Argentina}

En Argentina, una buena proporción de niños y niñas crecen en ambientes equipados con tecnologías y conectividad.

Según el Módulo de Acceso y Uso de Tecnologías de la Información y la Comunicación (MAUTIC) de la Encuesta Permanente de Hogares (EPH) que releva el Instituto Nacional de Estadísticas y Censos de Argentina (INDEC) para el cuarto trimestre 2018, el 63\% de los hogares tiene acceso a computadora y el 80.3\% tiene acceso a internet. En cuanto a la población de 4 años y más en el Gran Buenos Aires (donde se desarrolla nuestro estudio), el $82.9 \%$ usa telefonía celular, el 39.2\% computadora y el $76.3 \%$ utiliza internet ${ }^{2}$.

Otra fuente de datos reciente refiere a los accesos a internet registrados durante el cuarto trimestre del 2018. El INDEC sistematiza información provista por las empresas proveedoras de servicios de telecomunicaciones ${ }^{3}$. A nivel nacional se puede observar el sostenido crecimiento de accesos fijos y móviles en los últimos años. Según este mismo informe, aproximadamente la mitad de estos accesos se concentran en las jurisdicciones Ciudad de Buenos Aires y Provincia de Buenos Aires. 


\section{GRÁFICO 1}

Accesos a internet fijos y móviles. Enero 2016-diciembre 2018. Cobertura Nacional

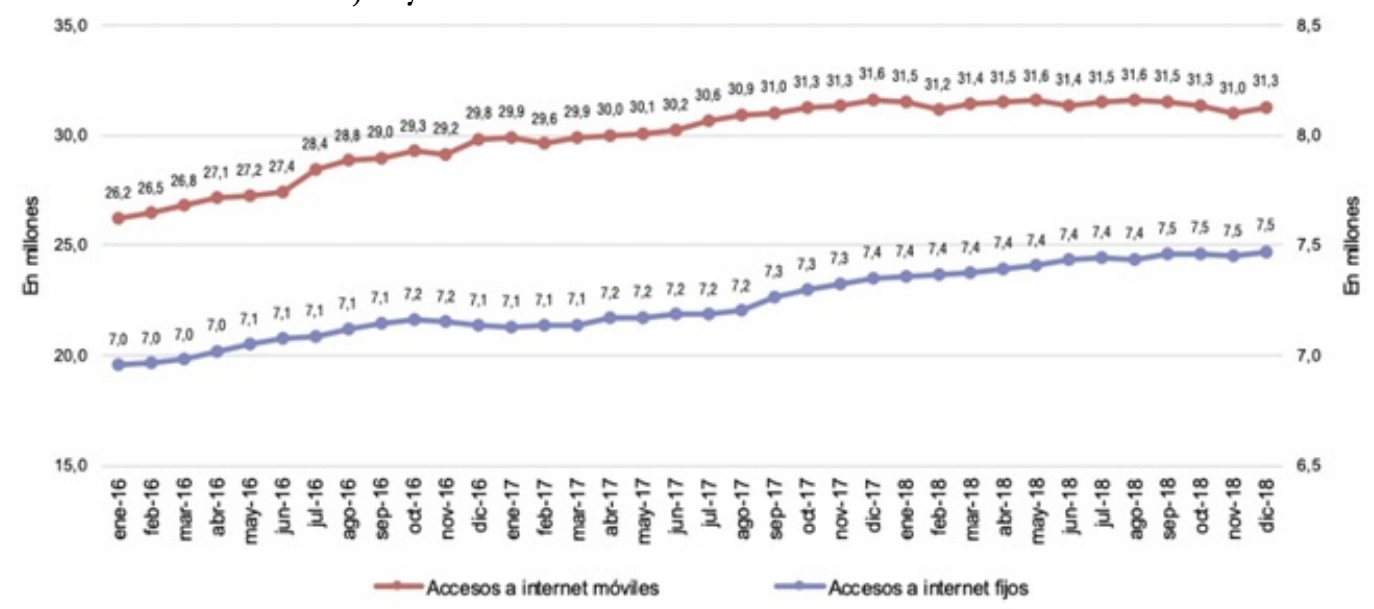

Fuente: Indec

Hay que tener en cuenta, no obstante, que según estos mismos datos aún quedaría casi un $20 \%$ de los hogares sin conectividad y un $23.7 \%$ de personas que no utiliza internet. Esos también son contextos en donde crecen niños y niñas. De acuerdo con el análisis que realiza Moyano (2019), en los hogares de nivel socioeconómico "alto" o "ABC1" (grupos familiares cuyo principal aportante tiene un perfil educativo universitario o "superior" y una elevada jerarquía ocupacional) la probabilidad de Acceso Restringido o No Acceso es 4\% (0.04) mientras que llega al 45\% (0.45) en el nivel socioeconómico “bajo" ("D”+"E”).

\section{3. ¿QUÉ USOS DE LAS TECNOLOGÍAS REALIZAN LXS CHICXS?}

La encuesta que realizamos en el UMI nos permite acceder al conocimiento que tienen las personas a cargo respecto de los usos de tecnologías que realizan lxs chicxs.

Según los adultos, los principales usos de las tecnologías que realizan niños y niñas de 6 a 8 años en el hogar están ligados a la conectividad (Internet y SmartTV) y los dispositivos móviles (teléfono celular y tablet). Como puede observarse en el Gráfico 2, los usos de Internet y teléfono celular lideran claramente superando el $80 \%$ y luego hay un segundo grupo de tecnologías integrada por Tablet (67\%), SmartTV (64\%) y TV por Cable o Satelital (62\%). El resto de las tecnologías se ubica por debajo del $50 \%$. 


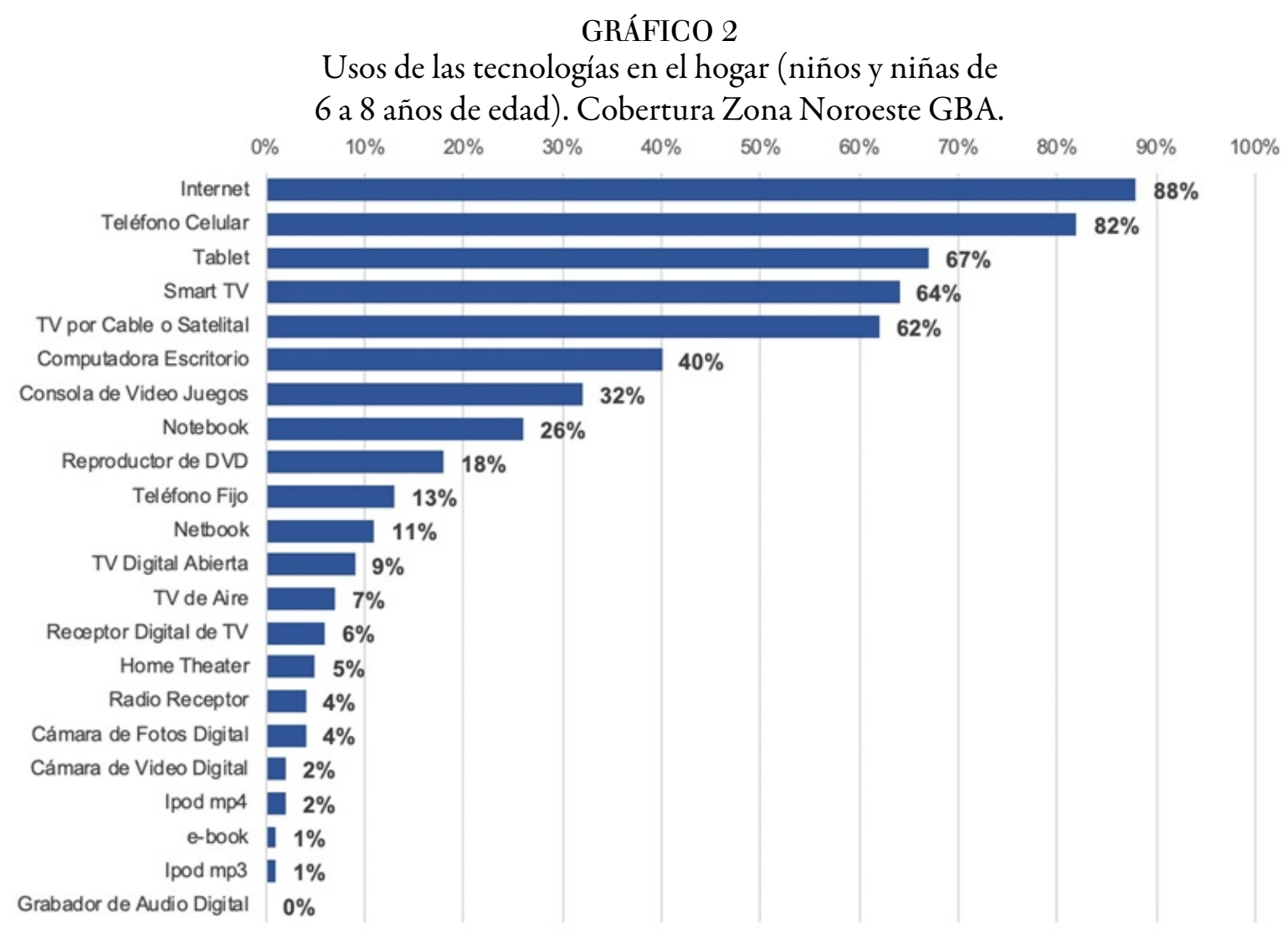

Fuente: elaboración propia a partir de Encuesta Sobre Apropiación de Tecnologías por parte de Niños y Niñas 2018 (UNGS/UMI)

El uso de Internet no solamente está ampliamente extendido en este segmento de niños y niñas sino que además es una tecnología con una frecuencia de uso absolutamente intensiva. Tal como se puede observar en el Gráfico 3, 3 de cada 4 de los niños y niñas que acceden a internet lo hacen con una periodicidad diaria y el $90 \%$ de ellos con una frecuencia de 4 veces por semana o superior. 


\section{GRÁFICO 3}

Frecuencia de uso de Internet (niños y niñas de 6 a 8 años). Cobertura Zona Noroeste GBA

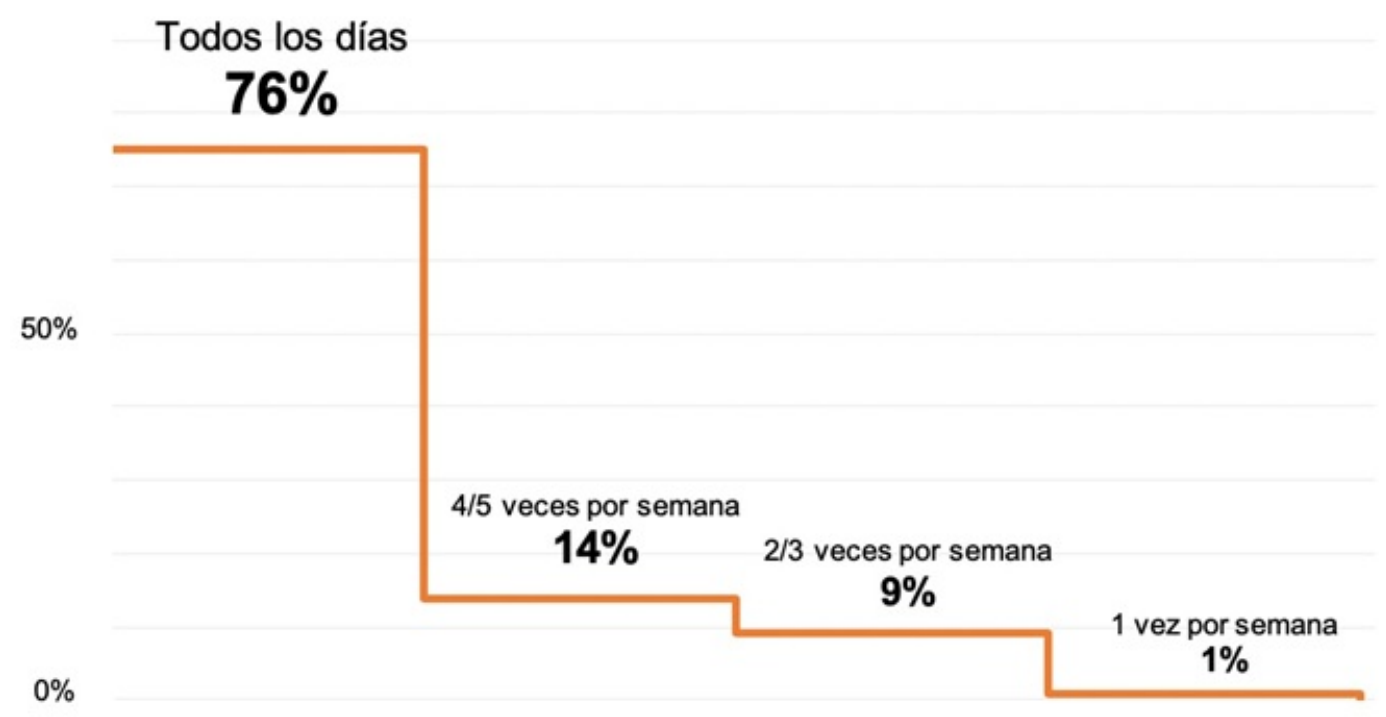

Fuente: Elaboración propia a partir de Encuesta Sobre Apropiación de Tecnologías por parte de Niños y Niñas 2018 (UNGS/UMI)

En promedio, los niños/as utilizan Internet con 2,7 dispositivos en el hogar. Como puede observarse en la Tabla 1, este valor promedio también presenta variaciones según la edad del niño/a, el tipo de escuela a la que asiste (gestión pública o privada) y al nivel socio-económico de su hogar, de manera tal que la cantidad de dispositivos aumenta sobre todo en el NSE alto

\begin{tabular}{|c|c|c|c|c|c|c|c|c|}
\hline \multicolumn{4}{|c|}{ TABLA 1: cantidad de dispositivos con los que accede a Internet. Cobertura Zone Noroeste GBA } \\
\hline \multicolumn{4}{|c|}{ EDAD DEL NIÑO/A } & \multicolumn{2}{c|}{ ESCUELA } & NIVEL SOCIO-ECONÓMICO \\
\hline TOTAL & EDAD 6 & EDAD 7 & EDAD 8 & PUBLICO & PRIVADA & $\begin{array}{c}\text { ALTO } \\
\text { (ABC1) }\end{array}$ & $\begin{array}{c}\text { MEDIO } \\
\text { (C2/C3) }\end{array}$ & $\begin{array}{c}\text { BAJO } \\
\text { (D1/D2E) }\end{array}$ \\
\hline $\mathbf{2 , 7}$ & 2,5 & 2,6 & 2,9 & 2,4 & 2,9 & 3,2 & 2,7 & 2,6 \\
\hline
\end{tabular}

Fuente: Elaboración propia a partir de Encuesta Sobre Apropiación de Tecnologías por parte de Niños y Niñas 2018 (UNGS/UMI)

El teléfono celular es el principal dispositivo con el que acceden a internet en el hogar con el $81 \%$. Luego aparece la Tablet con el 59\%, el SmartTV con el 43\% y la computadora de escritorio con el 34\%. En menor medida, la consola de videojuegos (17\%), notebook (16\%) y netbook (7\%).

El Gráfico 4 muestra que los chicos de 6 años usan el teléfono celular un poco más que los de 7 y 8 (que usan más variedad de dispositivos). 


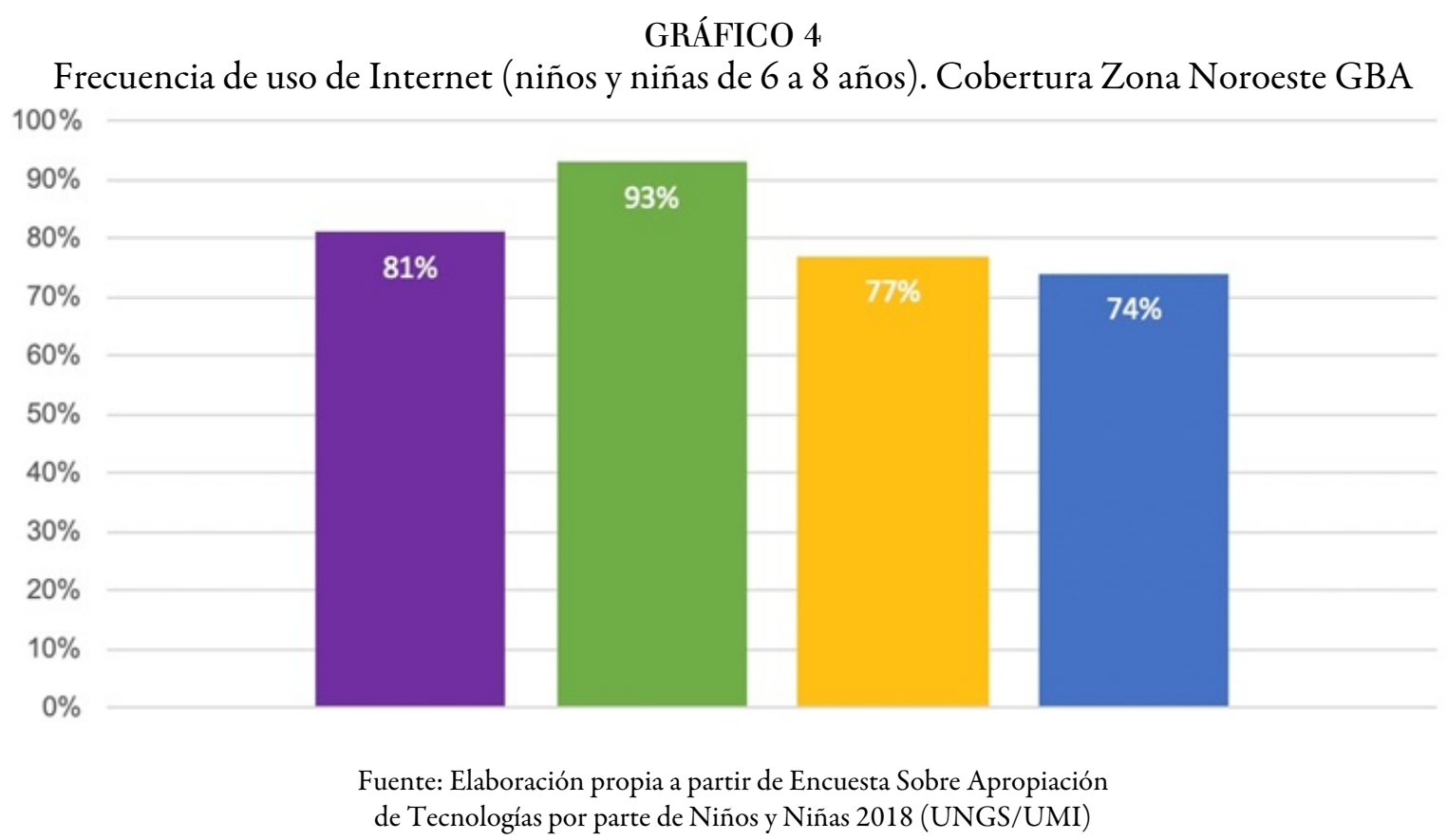

Youtube es la plataforma que lidera claramente los usos y apropiaciones de internet por parte de los niños/ as entre 6 y 8 años. Fue mencionado por prácticamente la totalidad de la muestra (99\%), tal como puede verse reflejado en el Gráfico 5.

En una fase previa de esta misma investigación se exploraron los usos de internet a través de entrevistas en profundidad, en las cuales las personas a cargo de niños y niñas también identificaron a Youtube en primer lugar. A través de las declaraciones de entrevistadas y entrevistados, se estableció que no se requiere la lectura y/o escritura para la realización de búsquedas. Los niños y niñas reconocen íconos, fotografías y otros indicadores. Utilizan además las órdenes orales y sus propios historiales.

Frente al predominio absoluto de Youtube, se destacan dos usos más: los videojuegos en línea (70\%) y escuchar música por Internet (62\%). El resto de los usos de internet fue mencionado por menos de la mitad de las y los entrevistas/os. 


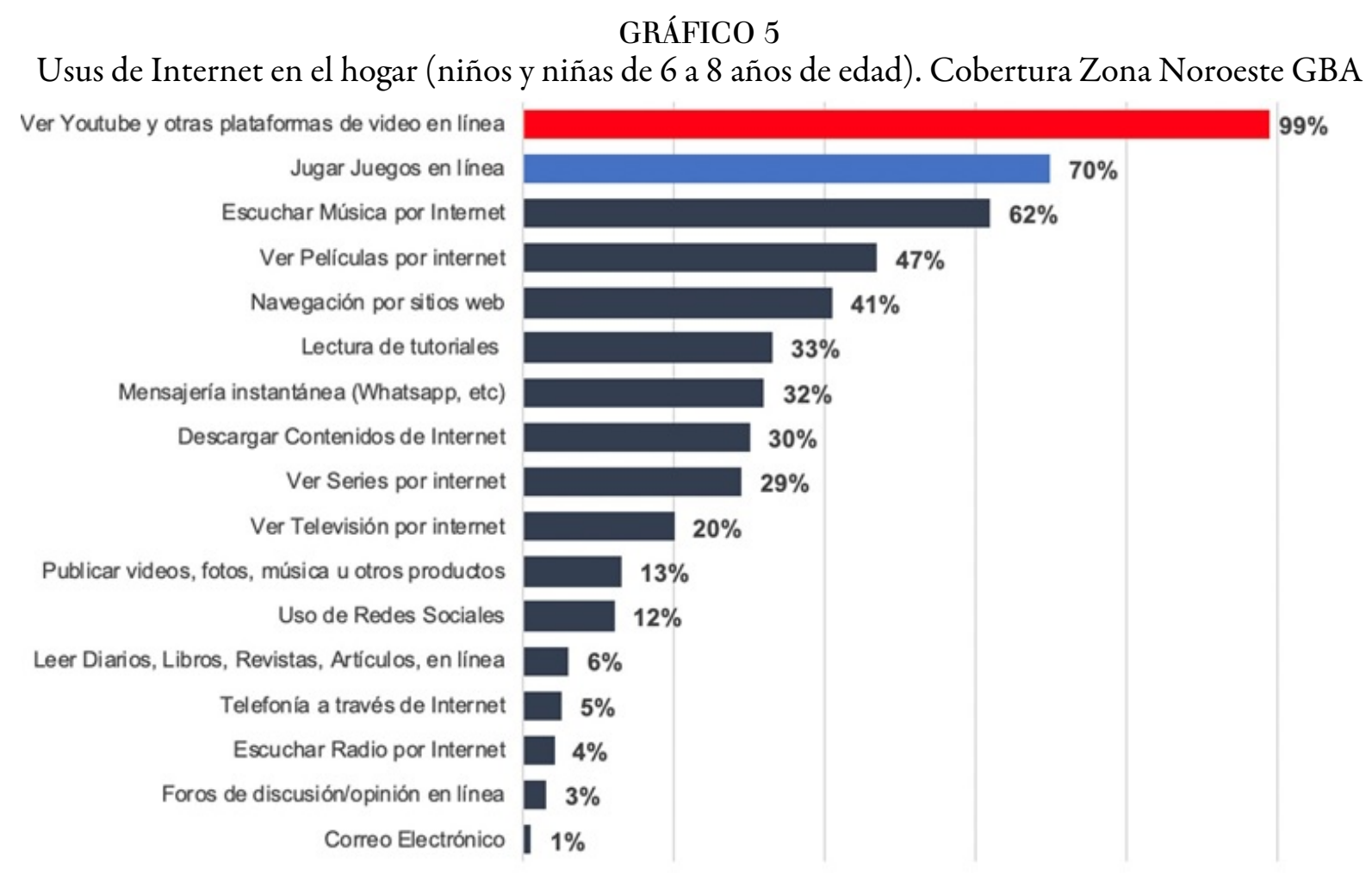

Fuente: Elaboración propia a partir de Encuesta sobre Apropiación de Tecnologías por parte de Niños y Niñas 2018 (UNGS/UMI)

En lo que respecta al segundo uso en importancia (juegos en línea), se observan diferencias por género. Entre los varones, el guarismo de uso asciende al $78 \%$ mientras que entre las mujeres se reduce al $62 \%$.

En los siguientes dos apartados se caracterizan los dos principales usos identificados: Youtube y juegos en línea. Se enfoca el tiempo dedicado, los principales dispositivos a partir de los cuales se accede a cada uno de ellos y se analizan los principales contenidos.

\subsection{Los usos De YouTUbe Y LOS JUEGos EN LÍNEA}

\subsubsection{El tiempo dedicado}

El principal uso de Internet de los niños y niñas de 6 a 8 años (Youtube) mencionado por el $99 \%$ de los adultos a su cargo, implica una dedicación en promedio de poco más de 2 horas y media por día (2.58 en horas). El Gráfico 6 muestra que el pico se produce por la tarde con un promedio de 1,28, es decir que prácticamente la mitad del tiempo total promedio se produce en ese momento del día. 


\section{GRÁFICO 6}

Horas Dedicadas a Youtube y Juegos en Línea (promedio en Horas). Cobertura Zona Noroeste GBA

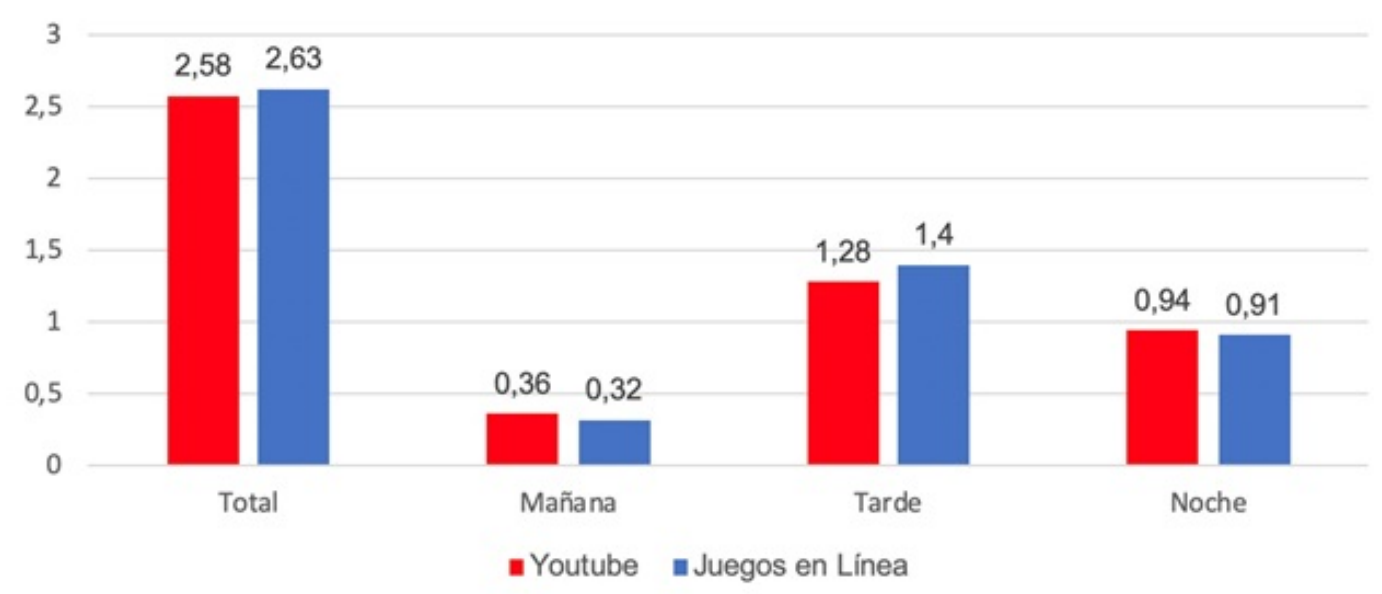

Fuente: Elaboració propia a partir de Encuesta Sobre Apropiación de Tecnologías por parte de Niños y Niñas 2018 (UNGS/UMI)

Un patrón similar en cuanto a la distribución del tiempo se da en los Juegos en Línea: la tarde presenta el promedio más elevado (1.40), con una leve diferencia sobre Youtube. En el resto de los momentos del día los valores promedios son muy parejos en respecto de la plataforma de video.

Comparando los distintos segmentos socio-demográficos se observa una diferencia a nivel de género. Los varones le dedican 2,8 horas promedio (llegando a superar la franja de las 4 horas diarias dedicadas a los Juegos en Línea), mientras que en el caso de las mujeres el tiempo promedio dedicado desciende levemente a 2,38.

\subsubsection{Los contenidos}

Los contenidos de Youtube más consumidos se presentan en el Gráfico 7. Los usos vinculados a la música y/ o la música infantil son los que lideran con el 35\%. Luego le siguen los Youtubers y los dibujitos animados con un $23 \%$ cada uno. Los juegos en línea o gamplays sin espeficar (12\%) y las películas/trailers de películas (11\%) también logran superar el 10\% de las menciones. 


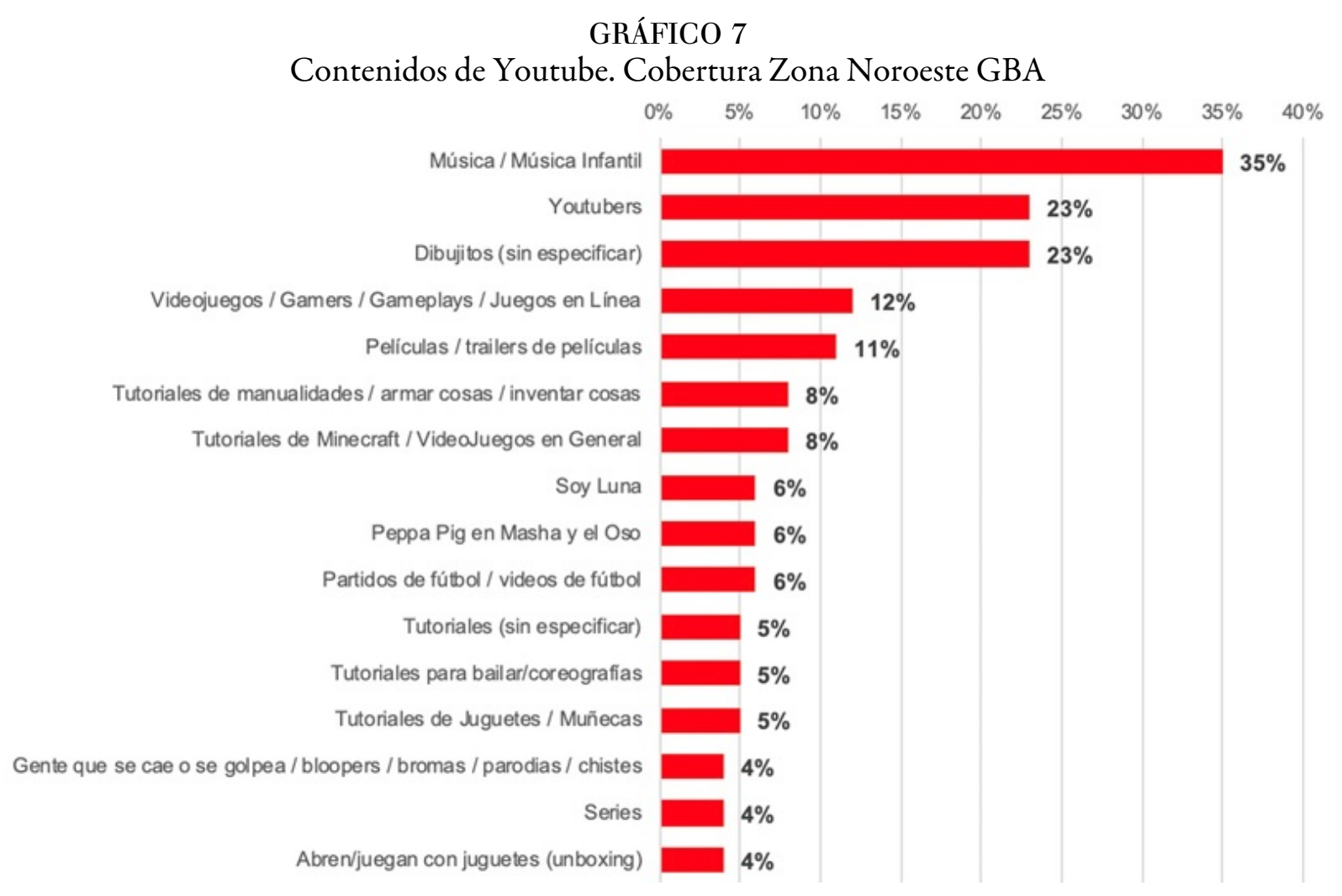

En relación a los Juegos en Línea, el Gráfico 8 muestra los 20 principales contenidos mencionados por los adultos a cargo de los niños. En particular, hay tres títulos que acumulan los porcentajes más importantes: Minecraft con el 16\% liderando el ranking, Fortnite con el 11\% y Roblox con el 9\%.

Sin especificar un título en particular, los adultos entrevistados también mencionaron géneros de juegos: los más importantes, aquellos que superaron el 10\%, son los de pelea/acción/guerra con el 16\%, de autos/de carreras con el $14 \%$ y los de cocina o aprender a cocinar con el $10 \%$ 


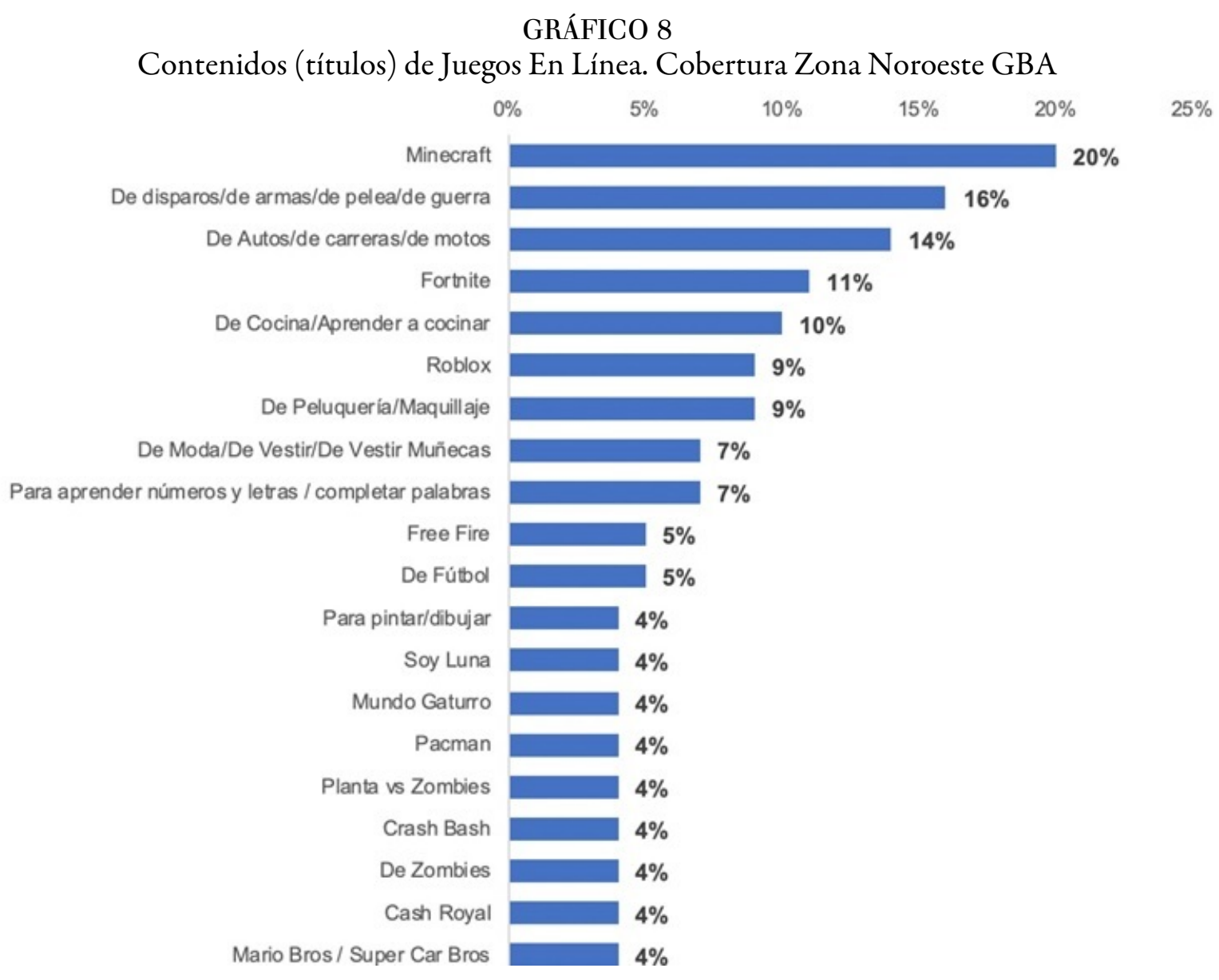

Fuente: Elaboración propia a partir de Encuesta Sobre Apropiación de Tecnologías por parte de Niños y Niñas 2018 (UNGD/UMI)

La encuesta establece que las preferencias varían por género. El Minecraft, los juegos vinculados al género de acción/pelea (entre ellos el Fortnite o el FreeFire) y los de fútbol, presentan porcentajes superiores entre los varones. Entre las mujeres, aumenta el porcentaje de los juegos vinculados a la cocina, peluquería, maquillaje y de moda o vestir (Ver Tabla 2). 


\begin{tabular}{|l|c|c|c|}
\hline \multirow{2}{*}{ TABLA 2: títulos o géneros de juegos en Línea según Género. Cobertura Zone Noroeste GBA } \\
\hline & \multirow{2}{*}{ TOTAL } & \multicolumn{2}{|c|}{ GÉNERO DEL NIÑO/A } \\
\cline { 3 - 4 } & & MUJER & VARON \\
\hline Minecraft & $20 \%$ & $10 \%$ & $\mathbf{2 9 \%}$ \\
\hline De disparos/de armas/de pelea/de guerra & $16 \%$ & $7 \%$ & $\mathbf{2 4 \%}$ \\
\hline De Autos/de carreras/de motos & $14 \%$ & $10 \%$ & $17 \%$ \\
\hline Fortnite & $11 \%$ & $5 \%$ & $\mathbf{1 7 \%}$ \\
\hline De Cocina/Aprender a cocinar & $10 \%$ & $19 \%$ & $2 \%$ \\
\hline Roblox & $9 \%$ & $7 \%$ & $12 \%$ \\
\hline De Peluqueria/Maquillaje & $9 \%$ & $13 \%$ & $5 \%$ \\
\hline De Moda/De Vestir/De Vestir Muñecas & $7 \%$ & $15 \%$ & - \\
\hline Para aprender números y letras / completar palabras & $7 \%$ & $10 \%$ & $4 \%$ \\
\hline Free Fire & $5 \%$ & $1 \%$ & $\mathbf{9} \%$ \\
\hline De Fútbol & $5 \%$ & $2 \%$ & $\mathbf{8} \%$ \\
\hline
\end{tabular}

Fuente: Elaboración propia a partir de Encuesta Sobre Apropiación de Tecnologías por parte de Niños y Niñas 2018 (UNGS/UMI)

Un aspecto que interesa especialmente es el consumo de tutoriales, ya que es uno de los que se asocia más directamente con la actividad de aprendizaje, en una modalidad autogestionada. Los tipos de tutoriales más consultados son los de manualidades y los de juegos en línea y alcanzan el $8 \%$ cada uno. Los niños consultan tutoriales relacionados con el consumo tecnológico, en particular videojuegos. Mientras que las niñas buscan aprender o imitar actividades ajenas a las tecnologías (como baile o cocina), tal como puede observarse en la Tabla 3.

\begin{tabular}{|l|c|c|c|}
\hline \multirow{2}{*}{ TABLA 3: cosas que ve habitualmente por Youtube (Zoom en Tutoriales). Cobertura Zona Noroeste GBA. } \\
\hline & TOTAL & GÉNERO DEL NIÑO/A \\
\cline { 3 - 4 } & $8 \%$ & $9 \%$ & $6 \%$ \\
\hline $\begin{array}{l}\text { Tutoriales de manualidades, armar o inventar cosas/ } \\
\text { reciclar }\end{array}$ & $8 \%$ & $3 \%$ & $\mathbf{1 3} \%$ \\
\hline $\begin{array}{l}\text { Tutoriales de Minecraft / de VideoJuegos en General / } \\
\text { De juegos en línea }\end{array}$ & $5 \%$ & $4 \%$ & $9 \%$ \\
\hline Tutoriales (sin especificar) & $5 \%$ & $\mathbf{9} \%$ & $0 \%$ \\
\hline Tutoriales para bailar/coreografías & $5 \%$ & $\mathbf{7} \%$ & $\mathbf{2} \%$ \\
\hline Tutoriales de Juguetes / Muñecas & $3 \%$ & $3 \%$ & $3 \%$ \\
\hline Tutoriales de Slime & $3 \%$ & $\mathbf{5} \%$ & $0 \%$ \\
\hline Tutoriales de cocina & $3 \%$ & $\mathbf{4} \%$ & $1 \%$ \\
\hline Tutoriales de maquillajes / de belleza & $1 \%$ & $1 \%$ & $0 \%$ \\
\hline Tutoriales de Yoga / Video de gimnasia & & & \\
\hline
\end{tabular}

\section{OBSERVACIONES SOBRE LOS CONSUMOS INFANTILES}

La encuesta revela los principales consumos que realizan niños y niñas de 6 a 8 años de edad, a través de internet, de acuerdo con las declaraciones de las personas adultas que están a su cargo. La información sintetizada en parágrafos anteriores permite realizar las siguientes observaciones: 
- El principal consumo corresponde a Youtube en donde destacan tres clases de productos que corresponden a otros tipos de medios de comunicación que convergen en esa plataforma: productos musicales, videos (de youtubers) y dibujos animados.

- La producción de contenidos por parte de los niños y niñas de este tramo de edad casi no se menciona en las entrevistas.

- Los tutoriales corresponden a un tipo de consumo más asociado con la actividad y el aprendizaje por parte de lxs chicxs. Se realiza sobre todo entre las niñas, aunque los niños también consultan algunos. El consumo de tutoriales está bastante asociado al resto de los usos y consumos de internet que se realizan por género.

- Los tipos de consumos que los niños y niñas realizan a través de Youtube no requieren competencias comunicativas ni tecnológicas más complejas que las involucradas en consumos de medios tradicionales. Como se refirió en apartados anteriores, no se requiere, por ejemplo, la lectura y/o escritura para la realización de búsquedas.

- El segundo uso de internet más importante es el de los juegos en línea, en donde adquiere relevancia la interactividad.

- El videojuego más jugado es el Minecraft (20\%), que se define como un videojuego de mundo abierto. Consiste en poner bloques para construir y recorrer un gran número de mundos y aventuras. Es uno de los juegos preferidos por niños y niñas, aunque sobre todo por los varones. Admite dos tipos de modos: el modo creativo, que permite a los jugadores crear y destruir fácilmente estructuras y mecanismos y también cambiar la música de fondo. El modo supervivencia, en el cual los jugadores disponen de recursos y deben realizar distintas acciones (construir estructuras, combatir obstáculos, administrar esfuerzos) para sobrevivir en distintos escenarios. Ambos tipos requieren una clase de participación por parte del jugador mucho más interactiva que en el caso de Youtube. Los usuarios se relacionan no solamente con la narrativa sino con todas las instancias en las que pueden intervenir para la producción de la misma, sus cambios y continuidades. Como hemos observado en talleres realizados con niños y niñas en el marco de la misma investigación, esto implica el desarrollo de un conjunto de competencias que hacen a la operación de la interfaz, la lectura de la imagen, la sincronización ojo-mano, el conocimiento de las pantallas, la orientación en el espacio digital para el seguimiento de los recorridos, entre otras.

El segundo videojuego más jugado es el Fortnite (11\%). Es interesante profundizar en la segunda etapa de investigación (más cualitativa) respecto de cuáles son los modos más jugados por los niños y niñas de estas edades. El juego presenta tres modos: Salvar el mundo, Battle Royale y Creativo. Más allá de que los tres usan el mismo motor y tienen recursos muy similares, incorporan los dos primeros la opción de jugar en forma cooperativa y el tercero ofrece la posibilidad de construir, agregar piezas, editar características, etc. Tanto la intervención en el entorno de juego a través de la construcción como la asociación con terceros para la participación colaborativa pueden representar modalidades de apropiación más complejas.

- De acuerdo con el relato de las personas adultas, las nenas prefieren videojuegos de cocina o que permitan aprender a cocinar. Existe una gran cantidad de juegos de este tipo, aunque no se distingue una gran variedad en lo que respecta a la solicitación que realizan respecto de la usuaria. El juego requiere hacer click en distintos componentes de la pantalla y el rango de complejidad es acotado: desde apilar capas de una torta, en el caso más simple, hasta incorporar los ingredientes de una receta, en los casos más sofisticados. Si bien aquí también se observa una clase de participación por parte de la jugadora un tanto más interactiva que en el caso de Youtube, el tipo de narrativa que se propone es sencilla, condicionada por el género "receta de cocina" y la participación en la producción de la misma es muy direccionada e instrumental. Las competencias requeridas están vinculadas sobre todo a la operación de una interfaz sencilla, la lectura de la imagen y la sincronización ojo-mano. A través de la 
aproximación cualitativa que se desarrollará en la próxima etapa de la investigación, nos proponemos indagar si se produce aprendizaje sobre las recetas o simplemente se resuelve el juego mecánicamente.

\section{Comentario de CIERre}

A través de esta encuesta hemos investigado distintos aspectos de los procesos de apropiación de tecnologías por parte de niños y niñas de 6 a 8 años de edad. En este artículo presentamos una caracterización de los usos que realizan, de acuerdo con las declaraciones de las personas adultas a cuyo cargo se encuentran.

A pesar de que el acceso a las tecnologías aumenta de manera sostenida, en Argentina hay una importante proporción de hogares que aún no tiene conectividad y en el Gran Buenos Aires hay una porción de la población que no usa internet, entre quienes cuentan niños y niñas. Esos hogares y esas personas quedan afuera de esta caracterización y de otras posibilidades asociadas con la conectividad. En consonancia con estudios realizados por el INDEC, nuestra encuesta revela que el Nivel Socio Económico y el clima educativo de los hogares son variables que condicionan el acceso y los usos de las tecnologías (en particular internet).

Es cierto que cada vez más chicxs acceden a distintos dispositivos y a internet a edades más tempranas y que les dedican una buena cantidad de tiempo. En un estudio sobre usos de televisión por cable realizado en 1997 establecimos que los entrevistados y entrevistadas (que tenían entre 6 y 12 años de edad) consumían un promedio de 3 horas diarias de televisión (Cabello, 2001). En la actualidad, ese tiempo parece estar destinado a internet. Sin embargo eso no implica directamente que se construya de manera casi "natural" y homogénea un sujeto más interactivo y menos receptor de contenidos. De acuerdo con los usos y consumos identificados a través de la encuesta se observa que lo que más se sostiene es, justamente, la posición de recepción de contenidos a través de un soporte diferente: la plataforma digital (Youtube). A los productos asociados a los medios de masas (videos y musicales) se suman otros típicamente propios de esa plataforma, como los videos de youtubers. Pero lejos de conllevar una posición interactiva, se consolida el consumo, tal como se demuestra en las preferencias por los gamers youtubers; hay niños y niñas que invierten más tiempo en mirar cómo esos personajes juegan que en jugar ellos mismos los videojuegos. Por otra parte, según los entrevistados/ as solamente el $13 \%$ de la muestra realiza actividades productoras de contenidos, como publicar videos, fotos, música u otros productos ${ }^{4}$. Si se toma en cuenta este dato puede introducirse una nueva pregunta respecto del modo como se desarrollan estas prácticas en contextos regionales diferentes. Aunque Trinidad y Zlachevsky (2013) tienden a relativizar ese aspecto, en España, Tur-Viñes, Núñez-Gómez, y GonzálezRío (2018) analizaron las prácticas de menores influyentes en Youtube (aunque no explicitan edades) y les atribuyen un estilo de gestión al que denominan "cuasiprofesional".

Pero en la zona del conurbano bonaerense donde desarrollamos nuestro estudio, es en los usos de videojuegos donde más se pone de manifiesto la interactividad, y ya hemos hecho referencia a una diferenciación por géneros. No nos hemos detenido en este artículo en el análisis de las implicancias que tienen las preferencias identificadas, desde el punto de vista de la construcción de sentido respecto de los roles, hábitos y representaciones asociadas a los géneros. Es sin duda una dimensión que retomaremos a través de la exploración cualitativa, para tratar de identificar y comprender el modo como los niños y niñas eligen y vivencian sus opciones y en qué medida y en qué sentido los relatos de los adultos expresan, efectivamente, esas opciones. Pero hemos visto que las preferencias atribuidas a las nenas (a partir de la identificación de los tipos de videos que consumen y, sobre todo, de los juegos que juegan), requieren competencias menos complejas y, en algún sentido, ofrecen menores oportunidades para desarrollar modalidades de apropiación más propias de las tecnologías interactivas. Por ejemplo, solamente un 3\% consulta tutoriales sobre juegos interactivos.

Avanzaremos en el análisis de estos temas, pero hemos presentado al menos un conjunto de variables que condiciona la relación que los niños y niñas establecen con las tecnologías digitales interactivas, en particular internet, buscando abonar a un análisis complejo que resista las posturas que tienden a naturalizar ese tipo de vínculos y a atribuirles ciertos atributos y propiedades, de manera reificante y directa. 


\section{REFERENCIAS}

Cabello, R (2001). Hijos de la TV por cable. Hacia un estudio longitudinal sobre los usos de la TV. Ponencia presentada en las Jornadas "50 años de Televisión en Argentina", organizadas por la Carrera de Comunicación Social de la Universidad de Buenos Aires. Ciudad Autónoma de Buenos Aires, Argentina.

INDEC (2019). Módulo de Acceso y Uso de Tecnologías de la Información y la Comunicación (MAUTIC).Encuesta Permanente de Hogares (EPH). Recuperado de: https://www.indec.gob.ar/uploads/informesdeprensa/mautic_ 05_19CF6C49F37A.pdf

INDEC (2019). Encuesta de Proveedores del Servicio de Accesos a Internet. Recuperado de: https://www.indec.gob.ar /uploads/informesdeprensa/internet_03_19.pdf

López, A. (2017). Apropiarse de la técnica: sobre la necesidad de estudiar los vínculos con las tecnologías desde una perspectiva sociohistórica. En R. Cabello A.López, A. (Edits) Contribuciones al estudio de procesos de apropiación de tecnologias (pp. 87-102) El Gato Gris y RIAT: Rada Tilly, Argentina

Moyano, R. (2019). Tecnologías y estructura social. Condiciones estructurales de inclusión digital en la población infantil de Argentina. Informe de avance de investigación. En Estudio de procesos de apropiación de tecnologias por parte de niños y niñas de 6 a 8 años de edad. UNGS. Mimeo.

Trinidad R. y Zlachevsky N. (2013). Jugar en contextos tecnológicos: uso y disfrute de Internet por niños y niñas de 8 a 10 años en Argentina, Paraguay y Perú. En Contratexto, No21.42. Recuperado de : https://www.researchgate.net/publication/321349470_Jugar_en_contextos_tecnologicos_uso_y_disfrute _de_Internet_por_ninos_y_ninas_de_8_a_10_anos_en_Argentina_Paraguay_y_Peru

Tur-Viñes, V., Núñez-Gómez, P. y González-Río, M. (2018). Menores influyentes en YouTube. Un espacio para la responsabilidad. En Revista Latina de Comunicación Social, 73, pp. 1211-1230. Recuperado de: http://www.re vistalatinacs.org/073paper/1303/62es.html

\section{Notas}

1 El clima educativo del hogar considera el promedio de los años de escolarización aprobados por los miembros del hogar mayores de 25 años de edad.

2 Ver Encuesta de Proveedores del Servicio de Accesos a Internet. Disponible en: https://www.indec.gob.ar/uploads/in formesdeprensa/internet_03_19.pdf

3 Ver Encuesta de Proveedores del Servicio de Accesos a Internet. Disponible en: https://www.indec.gob.ar/uploads/in formesdeprensa/internet_03_19.pdf

4 Cabe aclarar que en algunos casos esto es consecuencia de las regulaciones que establecen los padres u otras personas adultas a cargo del cuidado de los chicxs. 